\title{
EDITORIAL
}

\section{Obesity as a showcase for transdisciplinary research}

\author{
European Journal of Clinical Nutrition (2013) 67, 571-572; \\ doi:10.1038/ejcn.2013.72; published online 3 April 2013
}

Obesity as a showcase for trans-disciplinary research: Obesity is a rapidly growing public health challenge heading to be one of the main health problems in the world with high societal and individual costs. More so, severe obesity is a gateway to many other chronic diseases such as type- 2 diabetes, cardiovascular and heart diseases, and cancer, as well as to a multitude of social and psychological adverse conditions affecting quality of life, mental health, physical health and health-care costs as well as the efficiency of the workforce. We already know that to fully unravel the challenge of the obesity epidemic, we must take into account the obesogenic environment, the obese citizens and the way in which the society and individuals address obesity. Insights from Social Sciences and Humanities (SSH) will radically broaden the perspective on the obesity epidemic. The point of view of the obese, their rights and statuses as citizens, their life stories, narratives and so on. will be included, as well as questions about the impact of obesity discourse on the non-obese population and on other societal issues pertaining to history, social conditions, morality, law, esthetics, psychology and so forth.

In other words, to address obesity as a complex phenomenon, there is a need for integrating and mobilizing all relevant scientific disciplines building true trans-disciplinary research, which requires determination from all sides. We need to change and broaden our view on obesity by looking at the role of social structures, social inequality and stigma associated with obesity, the costeffectiveness of initiatives and interventions, and critically evaluate the potentials in choice architecture, behavior change and various forms of policy development, and political regulation. At the same time, we also need to heighten the awareness of societal effects and consequences of the obesity epidemic.

Sciences such as economics, anthropology, sociology, psychology, political sciences, architecture and urban planning, ethnology, philosophy, history, geography, communication and information sciences, science and technology studies, and many more disciplines within the SSH all hold a piece of this highly complex puzzle. By combining these scientific fields with nutritional and clinical research, physical activity, biomedical sciences and epidemiology, we will be able to answer new questions, and thereby secure new findings, solutions and greater impact in obesity research. Thus, the future potentials in obesity research aim at adding to our understanding of the complex system of the mechanism of obesity. The impact of such research includes identification of arenas for health promotion, prevention and policy making, and offers a spectrum of refined and personalized approaches to treatment, which balance the responsibility of the society and the autonomy of the citizens.

SSH contribution to tackle the obesity epidemic: On the 9-10th of January 2013, more than 50 researchers and stakeholders from around Europe gathered in Brussels for the workshop Social Sciences and Humanities contribution to tackle the Obesity Epidemic: Challenges and Potentials in Obesity Research towards Horizon 2020 (for more information on the workshop, please see www.foodfitnesspharma. ku.dk/ssh/; for more information on Horizon 2020, please visit http:// ec.europa.eu/research/horizon2020/index_en.cfm).
The workshop was initiated by the Danish SSH research community in the area of obesity research, and organized with the European Association for the Study of Obesity (EASO). It builds upon a movement of on-going European and national initiatives across Denmark, France and Germany, with the common objective of outlining obesity-related national research priorities and roadmaps.

The objectives of the workshop were to create a cross-European forum for identifying, describing and discussing future potentials in obesity research, to establish new and nurture existing networks and collaborations of researchers across SSH and the natural sciences with an interest in obesity research, and thereby to mobilize the significant European research capacities and potentials in preparing for Horizon 2020.

The main session was the round table discussions, which took its starting point in seven pre-defined themes. The themes built on some of the expectations and potentials for future obesity research, as previously identified by political stakeholders and researchers.

Delivering innovative research with a high societal impact: The participants were asked to identify future research potentials for each of the themes and to discuss what impact such research would have for addressing the obesity epidemic. Further, necessary scientific collaborators, interested stakeholders, and potentials, pitfalls and barriers were identified.

Through the round table discussions, it was clear that by combining the scientific disciplines embattling a societal challenge like obesity, we can pave the way for untapped and promising possibilities that can address obesity as a complex phenomenon. Some of the potential future research questions developed at the workshop were the following:

- How can we contribute to the creation of a robust common evidence base, by creating new tools, measurements and databases, enlarging the scope of assessing the extent and costs of obesity, costs and benefits of interventions and investments in prevention and treatment (including economy, as well as social, psychological and societal costs of obesity nationally and across Europe)?

- How do urban environments affect physical activity and food consumption (food availability and promotion, food outlets and supermarkets as part of the urban environment)?

- How can choice architecture and nudging be used effectively to change behavior?

- How can we effectively conduct follow-up research on interventions and programmes to make them more effective and inclusive by focusing on how treatment programmes are received and handled?

- How does body mass index function as a vehicle for stigmatization brought about by the health-care sector, by the community and media, by the industry and through selfstigmatization?

By strengthening new collaborations in obesity research from SSH across the natural sciences, we can enrich obesity and nutritional research. By expanding our common understanding and focusing on a broader spectrum of research questions we can cross scientific boarders and help unravel one of the great challenges for research today: making it possible to identify the key issues 
that will push our scientific frontiers to achieve the greatest social economic and societal impact for the citizens.

\section{ACKNOWLEDGEMENTS}

The making of the workshop and the original Executive Summary have been supported by funds from the Faculty of Health and Medical Sciences, University of Copenhagen and the Ministry of Science, Innovation and Higher Education in Denmark. Further, this workshop would not have happened without support from The European Association for the Study of Obesity (EASO), University of Copenhagen and creoDK.

Scientific Advisory Committee for the workshop: Professor Lotte Holm, University of Copenhagen; Professor Gema Frühbeck, University of Navarra, EASO Professor Peter Sandøe, University of Copenhagen; Professor Jean-Michel Oppert, University Pierre-et-Marie Curie, EASO; Professor Thorkild IA Sørensen,
University of Copenhagen and Institute of Preventive Medicine, Region $\mathrm{H}_{i}$ Professor John Blundell, University of London, EASO; Associate Professor Morten Ebbe Juul Nielsen, University of Copenhagen. Strategy and Organizing Committee for the workshop: Peter Børker Nielsen, Project Manager, University of Copenhagen; Anne Meidahl Petersen, EU Adviser, creoDK; Camilla Verdich, Research Coordinator, University of Copenhagen; Euan Woodward, Executive Director, EASO

L Holm, PB Nielsen, P Sandøe and MEJ Nielsen University of Copenhagen, Nørre Allé 20, Copenhagen, Denmark E-mail: pbnielsen@sund.ku.dk 